\title{
"Christianopolis" als Utopie der Bildung, Erziehung und Wissenschaft nach Johann Valentin Andreae (1586-1654)
}

Zusammenfassung: Eine Reihe utopischer Entwürfe im Denken des 17. Jahrhunderts akzentuiert die Bedeutung einer allen zugänglichen Bildung und Wissenschaft. Insbesondere im Leben und Werk Johann Valentin Andreaes (1586-1654) verbinden sich Kritik der gesellschaftlichen Verhältnisse mit dem Entwurf einer anderen und besseren Gesellschaft, wobei er wie so viele seiner Zeitgenossen den Staatstyp der aufgeklärten Herrschaft favorisiert. Wesentlich originärer sind dagegen jene Passagen seiner Beschreibung des Staates Christenstadt aus dem Jahr 1619, in denen sich Andreae mit der Wissenschaft und dem Bildungswesen beschäftigt. Ausführlich bestimmt er ihre Aufgaben sowie die Bedeutung und geht auf die Stellung der Wissenschaft in der Gesellschaft ein. Die in diesem Zusammenhang geäußerten Gedanken sind sowohl von philosophie- und wissenschaftshistorischem Interesse als auch unter aktuellen Gesichtspunkten aufschlussreich und stehen im Fokus des Artikels.

Schlüsselwörter: Christianopolis, Staat Christenstadt, Gesellschaftsutopie, Utopie der Bildung, Erziehung und Wissenschaft

Summary ("Christianopolis" as a Utopia of Education and Science according to Johann Valentin Andreae [1586-1654]):Education and sciences, that are accessible to all, is the focus of several complex and remarkable utopian visions from the 17th century. Particularly the life and work of Johann Valentin Andreae (1586-1654) shows the relation between the critique of social conditions and the idea of a better society. As many others at that time he favours a type of state marked as enlightened governance. However, his detailed description of the state "Christianopolis" from 1619, in which he addresses primarily the role of science and education in a society, shows significantly more independent concepts and implications. In his comprehensive explanation he specifies that science and education have certain responsibilities and importance for a better society. Those thoughts are meaningful and considerable both for philosophical and historical reflections of science and the vantage point of the present and shall therefore be the main focus of this article.

Keywords: Christianopolis, State Christen City, societal utopia, utopia of education, education and science

Резюме [(Аня Франц и Дитрих-Экхард Франц: „Christianopolis“ как утопия образования, воспитания и науки у Иоганна Валентина Андреа (1586-1654)]: Ряд утопических проектов философской мысли 17-го века акцентирует значение доступного образования и науки. В частности, в жизни и творчестве Иоганна Валентина Андреа (1586-1654) соединяются критика общественных отношений с планом другого, более лучшего общества, причем он, как $и$ большинство его современников, отдает предпочтение государственному типу просвещенного абсолютизма. Напротив, значительнее оригинальными являются те пассажи его описания государства Кристенштадт («Christianopolis»), датируемым 1619 годом, в которых Андреа занимается наукой и образованием. Он подробно определяет их задачи, а также значение и рассматривает положение науки в обществе. Выраженные в этой связи мысли являются показательными как с точки зрения философско-исторического и научно-исторического, так и с современных точек зрения и находятся в центре внимания статьи.

Ключевые слова: Christianopolis, государство Кристенштадт, общественная утопия, утопия образования, воспитания и науки 


\section{Einheit von Leben und Werk Andreaes}

Bildung und Wissenschaft spielen in den utopischen Gesellschaftsentwürfen des 17. Jahrhunderts eine besondere Rolle. Eingebettet in einen religiös-christlichen Kontext entwickeln zum Beispiel Johann Arndt (1555-1621) und Johann Valentin Andreae das sogenannte "wahre Christentum", in dem die Forderung zur Tat und zur Veränderung zur Lebensmaxime erhoben wird. Insbesondere im Leben und Werk Andreaes verbinden sich Kritik der gesellschaftlichen Verhältnisse mit dem Entwurf einer anderen, besseren Gesellschaft miteinander. Er schreibt seine Gedanken in der bekannten Veröffentlichung "Republicae Christianopolitanae descriptio" nieder, die im Jahre 1619 erstmals erscheint. ${ }^{i}$ Weiterführend zum Werk Andreaes seien hier besonders Beneš (1996), Kortmann (2007) sowie Wudmann (2008) erwähnt. Mit Sicherheit entsteht die „Beschreibung des Staates Christenstadt" unter dem Einfluss des von dem italienischen Philosophen Tommaso Campanella (1568-1639) verfassten "Civitas solis" (Der Sonnenstaat, 1602/1603), der zwar erst nach Erscheinen von Andreaes Werk veröffentlicht wird, den dieser aber wohl im Manuskript bereits kennt (vgl. Kirchenheim, 1892, S. 88).

Häufig wird Andreaes "Christianopolis" auch als kleinmütiges protestantisches Gegenstück zur weitaus bekannteren Utopie von Campanella hingestellt (vgl. ebd., S. 99f.; Kleinwächter, 1891, S. 55; Gysi et al., 1962, S. 244f.), wobei der Blick jedoch nicht genügend scharf auf die konkreten historischen Gegebenheiten gerichtet wird. Außerdem findet der Kontext seines Lebens und Schaffens oftmals nicht ausreichend Berücksichtigung. Schließlich ist es Johann Gottfried Herder (1744-1803), der sich um die Rettung und Verbreitung der Andreaeschen Gedanken verdient macht. Er sagt über ihn, dass man diesen Mann zuerst in seinem Leben kennen müsse, ehe man ihn in seinen Schriften kennenlerne (vgl. Herder, 1978, S. 150).

In der Tat bilden Leben und Werk dieses ungewöhnlichen Menschen eine untrennbare Einheit. Er ist nicht nur ein "Lehrer der echten Menschenliebe und Menschenweisheit" (ebd., S. 147), sondern nach übereinstimmenden Zeugnissen und Aussagen selbst in diesem Sinne als Reformer tätig. Gnadenlos und pointiert kritisiert er die Missstände seiner Zeit, allen voran die despotische Herrschaftsform in ihrer Ungerechtigkeit, Gewalt und Willkür. Beispielsweise beschreibt er in allegorischer Form den Triumphzug des Despotismus am Tage der Blutschuldenfeier des Menschheitsgeschlechts:

"Mord im Auge und Blut an der Faust schritten die treuen Diener des Despotismus einher. Manches Land von Europa würde erröthen, nannte ich die, welche mein Blick hier erkannte. Aber ich sah unter ihnen auch Männer aus einem Volke, das mir so theuer ist - laßt mich seufzen und schweigen." (Andreae, 1786, S. 81)

In diesem Zug gibt es zum einen edelmütige Gefangene und zum anderen würdelose Abhängige. Zu den in Fesseln geschlagenen Gefangenen zählt er Religion und Tugend, Freiheit und Edelsinn, Wohlsein und Selbstgefühl, Weisheit und Freimut. Zur zweiten Kategorie gehören die Günstlinge, Diener und Schergen. Sie verkörpern Machtanspruch, Gesetzlosigkeit, Gewohnheit und Gewalt. Auch die Schmeichler und Kuppler, die schlechten Journalisten und Dichter, die Sklavensinn, Intoleranz, Verstellung und Ränkesucht bedeuten, zählt er dazu. Mitten in diesem Zug der präsentiert sich der "Selbstherrscher":

"Sein Haupt, von Menschenblut trunken, wankte unter einer Krone aus Dolchspitzen, künstlich gefertigt. Der Thron, auf dem er sich brüstete, hatte die Gestalt eines Scheiterhaufens. Sein Gewand war das Gewand der Nacht, von Blutflecken furchtbar durchglänzt. Um ihn her lagen Todesurtheile, Lettres de cachet und Contributionslisten." (ebd., S. 82) 
Diese scharfsinnige Charakteristik despotischer Machtverhältnisse verlangt eine gehörige Portion Mut und es gab wenige, die den Mut aufbrachten, solche Wahrheiten in aller Öffentlichkeit auszusprechen. Johann Valentin Andreae zählt zu diesen Mutigen. In seinen Schriften übt er harsche Kritik an Politik und Lebensweise an den Fürstenhöfen, an der orthodoxen, lebensfremden Theologie und deren Vertretern, und vor allem an den katastrophalen Bildungsverhältnissen. "Ob wir die Kirchen, die Herrscherhäuser, die Bildungsanstalten betrachten - nirgends fehlen die alles beherrschenden Laster wie Ehrgeiz, Habsucht, Völlerei, Wollust, Neid, Faulheit", resümierte er in der Einleitung zu "Christianopolis" (1977, S. 7).

\section{Der Gesellschaftskritiker Andreae}

Eine Veränderung dieser Zustände herbeizuführen, war sein oberstes Ziel. Dabei lassen sich im Wesentlichen drei Etappen im Wirken Andreaes unterscheiden: Kritik der bestehenden Verhältnisse, Entwurf einer neuen Gesellschaftsform, Umsetzung der damit verbundenen Reformideen.

Die erste Etappe ist die bereits erwähnte Unzufriedenheit mit den bestehenden Verhältnissen, wobei der allgemeine "Sittenverfall" vor allem am Beispiel des Staates, von Religion und Kirche sowie von Wissenschaft, Bildung und Literatur dargestellt wird. Hier sind in erster Linie der "Menippus" (1617), eine Dialogfolge über verschiedenste Zeiterscheinungen, und die "Mythologia Christiana" (1619), eine ebenfalls lateinisch verfasste Sammlung von Erzählungen und Gleichnissen mit emblematischem Charakter zu nennen. Bereits 1616 waren zwei Schriften erschienen, die gewissermaßen den Auftakt zu dieser Gruppe gaben, nämlich "Turbo sive moleste et frustra per cuncta divagans ingenium" und "Chymische Hochzeit: Christiani Rosenkreutz anno 1549". Mit Hilfe unterschiedlicher literarischer Formen, einmal als Schauspiel, zum anderen als mystisch-satirische Erzählung - setzt er sich kritisch mit den Zeitverhältnissen auseinander. Zugleich zeichnet sich bereits in dieser Etappe das Verfolgen eines pansophischen Ideals und das Bemühen um die Durchsetzung des "wahren Christentums" ab.

Vollendete Gestalt erlangen sie jedoch erst in der "Republicae Christianopolitanae descriptio", jener Utopie eines christlichen Staates, die den Mittelpunkt der zweiten Etappe bildet. Nunmehr wird der kritischen Darstellung der Mängel eine mögliche gesellschaftliche Alternative gegenübergestellt.

Von der äußeren Form her gleicht Andreaes utopischer Entwurf "Christianopolis" vorangegangenen und auch nachfolgenden Utopien. Auf die Verbindung mit Campanellas "Civitas solis" wurde hingewiesen. Dennoch handelt es sich um eine bemerkenswerte eigene, originäre Leistung, denn Andreae reflektiert die deutschen Verhältnisse, und seine Beschreibung des idealen Staatswesens ist ein Kontrast zu dem, was seiner Meinung nach in deutschen Landen der Veränderung bedürfte. Deshalb durchzieht sein Werk von Anfang bis zum Ende die Gegenüberstellung von Kritikwürdigem und Veränderbarem. Viel deutlicher als anderswo zeigt sich gerade in Andreaes "Christianopolis" der Bezug zur gesellschaftlichen Realität.

Natürlich geht es auch hier um das in vielen Staats-Utopien verwendete Motiv der Insel. Nachdem der schiffbrüchige Fremde gerettet und verschiedenen Prüfungen unterzogen wurde, findet er Einlass in die Stadt, deren Einrichtungen sodann im Einzelnen beschrieben werden.

Die Produktion in dem vorgestellten Gemeinwesen beruht vornehmlich auf der Landwirtschaft, auf Ackerbau und Viehzucht. Daneben gibt es eine Reihe verarbeitender Betriebe, wie z. B. Mühlen, Backund Schlachthäuser, Keltereien, aber auch mechanische Werkstätten, namentlich zur Holzverarbeitung und Papierherstellung. Die Produktion erfolgt nach vorheriger Planung und alle Erzeugnisse, sowohl die der Landwirtschaft als auch die von Industrie und Handwerk werden 
zunächst in ein öffentliches Lager geliefert, von wo aus sie, den Bedürfnissen entsprechend, gerecht verteilt werden. Durch den Fleiß und die Geschicklichkeit der Einwohner, aber gleichzeitig auch durch den Verzicht auf Luxus und Verschwendung existiert ein Überfluss an allem Lebensnotwendigen. Zudem kann

"man eine gerechte Form des Warenaustauschs erleben, die nicht auf den Profit, sondern auf ein vielseitiges Angebot ausgerichtet ist. Auf diese Weise haben wir die besonderen Segnungen eines jeden Landstrichs vor Augen und sehen sie im Austausch miteinander, so daß wir an einem Ort gewissermaßen an der gesamten Erde Anteil haben." (Andreae, 1977, S. 25f.).

Geld existiert in dieser Gesellschaft zumindest für den persönlichen Gebrauch nicht. Wozu sollte es auch gut sein, wenn jeder ohnedies erhält, was er zum Leben benötigt? So konstatiert er verbittert:

"Alles ist für Geld zu haben, und fast nur für Geld. Für Geld feil ist die Religion und Gerechtigkeit, für Geld die Würden und der Anspruch auf Würden, Kenntnisse, Liebe, Freundschaft, Macht, Freiheit, Adel, Gleichheit, und Leben - alles um Gold!" (Andreae, 1786, S. $77)^{\mathrm{ii}}$

Der Lohn für die geleistete Arbeit ist vorwiegend ein moralischer, wobei jeder einzelne mit seiner Arbeit letztlich auch zum eigenen Unterhalt beiträgt. Vor allem aber zählen die hohe Achtung durch die Mitbürger, die Vorbildfunktion gegenüber der Jugend, die Anerkennung durch Hervorhebung, aber auch das „süße Bewusstsein eines redlichen Gewissens“, „die Weite eines erleuchteten Geistes“ oder die „Würde der Selbstbeherrschung“ (Andreae, 1977, S. 36).

Die Arbeit erfährt, ähnlich wie bereits bei Thomas Morus (1478-1535) und Campanella, eine hohe Wertschätzung; sie ist Pflicht und Ehre zugleich. Die Arbeitszeit umfasst nur wenige Stunden pro Tag. Sie ist aber äußerst produktiv, weil sie maximal ausgenutzt wird, die Arbeit mit Fach- und Sachkenntnis ausgeübt und auf ein ausgewogenes Verhältnis von Arbeit und Erholung geachtet wird:

"Dennoch wird nicht weniger geleistet als anderswo, denn Nichtstun über die erlaubte Zeit hinaus gilt allen als Schande. Während sonst zehn Arbeitende einen Faulenzer ernähren, ist es nur verständlich, daß von allen hiesigen Arbeitern jeder einzelne mehr Freiheit behält, als er unter anderen Bedingungen hätte." (ebd., S. 34)

Zugleich ist sie ständig mit der Forderung nach Schöpfertum und mit der Einführung wissenschaftlich-technischer Neuerungen und Erfindungen verbunden. Nicht zuletzt trägt dazu bei, dass die Arbeit ihren Charakter als Last verliert.

"Wo keine Knechtschaft ist, empfindet der Mensch auch keinen Widerwillen gegen die Anstrengung, sie belastet und entnervt ihn nicht. Wer wollte bezweifeln, daß [...] sich die Kräfte und der Fleiß vervielfachen und alles leichter und besser gerät, als wenn man [...] sinnlose Lasten aufeinandertürmt." (ebd., S. 34)

Deshalb wird Arbeit auch nicht nur "nach den Erfordernissen der Notwendigkeit" geleistet, sie ist zugleich und darüber hinaus Mittel zur Entäußerung und Selbstverwirklichung des Menschen. Arbeit wird entsprechend „als ein Wettstreit von Kunsthandwerkern, damit der menschliche Geist die Möglichkeit findet, sich mit verschiedenen Werkzeugen Ausdruck zu geben" (ebd., S. 31) dargestellt.

Weit weniger spektakulär ist die Schilderung der staatlichen Ordnung, in der er der Aristokratie als Regierungsform deshalb den Vorzug gibt, weil sie die beste Gewähr gegen die Tyrannei biete. Es regiert ein Dreierrat, bestehend aus einem Priester, einem Richter und einem Gelehrten. Ihnen zur 
Seite steht ein unmittelbarer Helfer, der als Diakon bzw. als Wirtschaftsverwalter beschrieben wird. Ihre Ehefrauen werden in allegorischer Weise als Gewissen, Vernunft oder Wahrheit gezeichnet.

Weiterhin stützt sich der Dreierrat jeweils auf einen Senat, der in Fachangelegenheiten berät. Wichtige Entscheidungen werden in einer gemeinsamen Versammlung der Vorsteher und Senate getroffen. Frömmigkeit, Klugheit und Erfahrenheit sind die wichtigsten Kriterien für die Übertragung solch hoher Ämter. Sehr akzentuiert wird festgestellt, dass es in diesem Staatswesen keine Würde, kein Ansehen um der Herkunft willen gibt, desgleichen wird auf die Vorbildwirkung und auf die väterliche Art zu regieren hingewiesen. Andreae favorisiert hier eindeutig den Staatstyp der aufgeklärten Herrschaft wie so viele seiner Zeitgenossen.

\section{Der Bildungsexperte Andreae}

Wesentlich interessanter und originärer sind dagegen jene Passagen, in denen sich Andreae mit der Wissenschaft und dem Bildungswesen beschäftigt. Ausführlich bestimmt er ihre Aufgaben sowie ihre Bedeutung und geht auf die Stellung der Wissenschaft in der Gesellschaft ein. Die in diesem Zusammenhang geäußerten Gedanken sind sowohl von philosophie- und wissenschaftshistorischem Interesse als auch unter aktuellen Gesichtspunkten aufschlussreich. "Die Wissenschaftskrämer haben viel zusammengeschwatzt", merkt er bereits in der Einleitung an, "um ihre Unwissenheit, ihre mangelnde Sprachkenntnis, die Käuflichkeit akademischer Grade, die unersättliche Geldgier zu verteidigen - zum äußersten Unwillen der wahren Gelehrsamkeit" (ebd., S. 8). Andreae stellt im Einzelnen anhand von acht Hörsälen folgende Disziplinen vor: Grammatik, Dialektik, Arithmetik, Musik, Astronomie, Physik, Ethik und Theologie. Ein weiteres Kapitel außerhalb der Hörsäle widmet er der Malerei.

In seinen Untersuchungen fallen zunächst zwei Momente besonders auf, einmal der enge Zusammenhang zwischen wissenschaftlicher Erkenntnis und deren praktischer Nutzung. Zum anderen betont er die Notwendigkeit einer breiten Vermittlung der Erkenntnisse und Erfindungen der Wissenschaft in sogenannten "Schausammlungen". Der Wissenschaft soll also ihr apartes Dasein entzogen werden, sie soll allen zugänglich sein und damit für alle verständlich werden. Das soll Wissenschaft vorrangig deshalb, weil Andreae einen engen Zusammenhang zwischen Arbeit und Wissenschaft sieht. Wenn man erfolgreich und mit Freude an der Arbeit produzieren will, muss man nicht nur die handwerklichen Fähigkeiten und Techniken beherrschen, man muss ebenso mit dem Material vertraut sein, mit dem man umgeht, das man bearbeiten soll und will. In seinem "Christianopolis" werden die Menschen

"nicht wie Arbeitstiere zu Tätigkeiten gezwungen, in die sie keinen Einblick bekommen, sondern sie erhalten vorher eine eingehende Schulung in die Naturkunde und haben ihre Freude an der Arbeit mit dem Naturmaterial." (ebd., S. 26)

Es werden jedoch nicht nur spezielle Kenntnisse vermittelt, sondern auf eine hohe Allgemeinbildung und Grundlagenkenntnisse Wert gelegt: "Man meint aber, dir noch nichts erklärt oder gezeigt zu haben, wenn man dir nicht auch die ursächlichen Zusammenhänge und den Bauplan der gesamten Welt deutlich gemacht hat." (ebd., S. 26f.).

Aufgabe der Wissenschaft ist es, dafür zu sorgen, "das in der Theorie Erreichte durch die Praxis zu bestätigen und nicht nur die Bezeichnungen der Dinge, sondern die Dinge selbst zu kennen" (ebd., S. 73). Die Natur selbst ist der Ausgangspunkt, es gilt, die Geheimnisse, die die Erde verborgen hält, wiederzuentdecken, sie zu erforschen "zum Nutzen der Menschen und zu Heilzwecken" (ebd., S. 71). 
Bezeichnend ist in diesem Zusammenhang, dass der Komplex der von ihm hier beschriebenen Wissenschaften - mit Ausnahme der Malerei - nur naturwissenschaftlich-mathematische Disziplinen enthält, wobei die Malerei allerdings auch vor allem wegen ihrer praktischen Momente und Tendenzen (Architektur, Optik, Festungsbau, technisches Zeichnen und Entwürfe für Maschinen) Aufnahme gefunden hat.

Andere Fachgebiete, wie etwa die Geschichte, Jurisprudenz, Ethik usw., sind für ihn weniger Gegenstand der Forschung, als vielmehr der Ausbildung, wie überhaupt diese beiden Gesichtspunkte sehr voneinander geschieden sind. Während es bei der Bildung und Erziehung, die einen hohen Stellenwert im Gesamtsystem einnehmen und von ihm in ihrer Einheit als "höchst wichtige Aufgabe" (ebd., S. 80) bezeichnet werden, darauf ankommt, nützliches Wissen anwendungsbereit zu vermitteln, hat die Forschung die Aufgabe, nicht nur tiefer in die Geheimnisse der Natur einzudringen und neue, tiefere Kenntnisse zu erwerben, sondern alles bisherige Wissen kritisch zu überprüfen. Er bemerkte diesbezüglich:

"Was durch den Fleiß früherer Geschlechter aus dem Brunnen der Natur emporgebracht und herausgezogen worden ist, wird überprüft, damit wir erfahren, ob ihre Eröffnungen richtig und zuverlässig sind." (ebd., S. 72)

Andreae ruft hier also zum Zweifel auf, aber nicht in skeptizistisch-negierenden Sinne, sondern als Methode zur tieferen Erkenntnis. Er reiht sich damit in jene Linie der progressiven skeptischen Philosophie ein, die von Michel de Montaigne (1533-1592) zu Pierre Bayle (1647-1706) führt und die auf die deutsche Aufklärung einen großen Einfluss ausübte. Das, was bei ihm in Ansätzen gefordert wurde, einen Geist des exakten und zugleich kritischen Denkens, hat sein Zeitgenosse René Descartes (1596-1650) als Grundprinzip seiner Methodenlehre formuliert.

\section{Andreae als Reformer}

So stellt sich Johann Valentin Andreae schließlich nicht als provinzieller Theologe dar, wie er nur allzu oft präsentiert wird, sondern er blühte "in seinem Jahrhundert wie eine Rose unter Dornen" (Herder, 1978, S. 147), um noch einmal Herders Hochachtung für diesen Menschen sprechen zu lassen.

Wie recht er mit dieser Einschätzung hatte, wird auch deutlich, wenn abschließend ein Blick auf die dritte Etappe des Lebens und Schaffens von Johann Valentin Andreae geworfen wird, die durch das unermüdliche Wirken für Reformen sowohl des kirchlichen wie des öffentlichen Lebens, vor allem des Schulwesens, gekennzeichnet ist. Nunmehr bemüht er sich, das, was er als richtig, wichtig und notwendig erkannt hat, umzusetzen. Insbesondere seine Tätigkeit in Calw, wohin er als Superintendent berufen wird und wo er fast zwanzig Jahre wirkt, zeigt Andreae als einen Philanthropen, der sich auf vielfältigste Weise für das Bessere einsetzt. Hervorzuheben ist in diesem Zusammenhang die Gründung des "Färberstifts", einer Stiftung, die es sich zur Aufgabe macht, Handwerker und Lehrlinge zu fördern, bedürftige Studenten zu unterstützen, aber ebenso Kranke zu pflegen sowie (Waisen-) Kinder aufzuziehen und auszubilden. Mittel dieser Stiftung dienen ferner dazu, eine Stadtbibliothek einzurichten.

Die Auswirkungen des Dreißigjährigen Krieges mit seinen Zerstörungen, Hungersnöten und Krankheiten bemüht er sich zu mildern und in Grenzen zu halten und selbstverständlich richtet er als Theologe besonderes Augenmerk auf die Reformierung innerkirchlicher Einrichtungen und des geistlichen Lebens überhaupt. Ganz besondere Aufmerksamkeit allerdings verdient seine Wirksamkeit auf dem Gebiet des Schulwesens. Schon in den zwanziger Jahren des 17. Jahrhunderts verfasst er die Schrift "Theophilus oder Ratschläge über die sorgfältige Pflege der Religion, die 
bessere Einrichtung des Lebens und die vernünftige Gestaltung des Unterrichts", die zunächst jedoch nicht erscheinen darf, wie er überhaupt wegen seiner Ideen zunehmend der Ketzerei verdächtigt wird.

Ebenso wie sein Zeitgenosse, der bedeutende progressive Pädagoge Wolfgang Ratke (1571-1635), tritt er für eine Erziehungsreform im Zusammenhang mit einer Reform der Gesellschaft ein. Beide gehen von dem Prinzip einer allgemeinen Bildung aus und bemühen sich um die Einführung fortschrittlicher Unterrichtsmethoden. Andreae betont die Einheit von Unterweisung und Erziehung und bezeichnet als Ziel der Ausbildung die "Erhaltung des Gemeinwesens und die Ausrichtung auf das künftige Leben" (Andreae, 1977, S. 81).

Die von ihm in "Christianopolis" dargelegten Auffassungen über das Schulwesen finden Zustimmung und Anerkennung durch den bedeutenden tschechischen Pädagogen und Philosophen Jan Amos Komenský (1592-1670), mit dem Andreae einen regen Briefwechsel unterhält. Einziger, aber nicht unbedeutender Erfolg seines unermüdlichen Wirkens auf diesem Gebiet war die Einführung der Schulpflicht in Württemberg im Jahre 1648, ansonsten stößt er jedoch, ebenso wie Ratke in AnhaltKöthen, an Grenzen, die in dieser Zeit nicht zu überwinden sind.

Als Andreae 1644 Mitglied der Fruchtbringenden Gesellschaft, einer Vereinigung zur Förderung der deutschen Sprache, wird, gibt er sich den bezeichnenden Namen "Der Mürbe". Er lässt nicht ab, gegen Despotismus, Sophisterei und Heuchelei, vor allem aber gegen die Unwissenheit, die er als Quelle aller Übel ansieht, anzukämpfen. Sein theoretisches und praktisches Wirken wird in der Folge von der Aufklärungsbewegung aufgenommen und weiter entwickelt. Doch nicht nur als Wegbereiter der späteren Aufklärung ist Andreaes Wirken von großer Bedeutung, sondern ebenso im Hinblick auf heutige Herausforderungen im Bildungsbereich. Angesichts der immer größer werdenden Komplexität des Wissens stellt sich auch heute wieder die Zugangsfrage zu dem weltweit verfügbaren Wissen. Allerdings auf einer anderen Ebene: Digitale Plattformen, Apps, unvorstellbare Speichermöglichkeiten ermöglichen einen unbeschränkten Zugang zu Wissen, aber sie sind kein Garant für Bildung. Bildung bedeutet im Sinne Andreaes nützliches Wissen anwendungsbereit zu vermitteln und da gilt es wie vor 400 Jahren neue Wege zu beschreiten.

\section{Literatur}

Andreae, J.V. (1619): Republicae Christianopolitanae Descriptio. Argentorati [Strasbourg]: Sumptibus haeredum Lazari Zetzneri.

Andreae, J.V. (1786): Dichtungen zur Beherzigung unseres Zeitalters. Mit einer Vorrede von Johann Gottfried Herder. Leipzig: Göschen.

Andreae, J.V. (1799): Selbstbiographie. Aus dem Manuscripte übers. u. m. Anm. begl. v. D. C. Seybold. Winterthur: Steiner.

Andreae, J.V. (1977): Christianopolis. Utopie eines christlichen Staates aus dem Jahre 1619. Leipzig: Koehler \& Amelang.

Beneš, J. (1996): J.V. Andrae (1586-1654) - Erneuerer der christlichen Erziehung. In: Golz, R./Mayrhofer, W. (Hrsg.): Luther und Melanchthon im Bildungsdenken Mittel- und Osteuropas. Münster: LIT-Verlag, S. 155-160.

Biesterfeld, W. (Hg.) (1975): J. V. Andreae: Christianopolis. Aus dem Lateinischen übersetzt, kommentiert und mit einem Nachwort v. Wolfgang Biesterfeld. Stuttgart: Reclam-Verlag.

Brecht, M. (1977): Johann Valentin Andreae. Weg und Programm eines Reformers zwischen Reformation und Moderne. In: Brecht, M. (Hg.): Theologen und Theologie an der Universität Tübingen. Beiträge zur Geschichte der Evangelisch-Theologischen Fakultät. Tübingen: Steiner: S. $270-343$. 
Dülmen, R.V. (1978): Die Utopie einer christlichen Gesellschaft. Johann Valentin Andreae (1586 - 1854). Stuttgart: Frommann-Holzboog.

Gysi, K./Böttcher, K./Albrecht, G./Krohn, P.G. (Hg.) (1962): Geschichte der deutschen Literatur von den Anfängen bis zur Gegenwart. Bd. 5: 1600 - 1700. Berlin: Volk und Wissen.

Herder, J.G. (1978): Denkmale und Rettungen. Berlin/Weimar: DDR Verlag.

Hoßbach, W. (1978): Johann Valentin Andreä und sein Zeitalter. Leipzig: Reimer.

Kirchenheim, A.v. (Hrsg.) (1892): Schlaraffia politica. Geschichte der Dichtungen vom besten Staate. Leipzig: Grunow.

Kleinwächter, F. (1891): Die Staatsromane. Ein Beitrag zur Lehre vom Communismus und Socialismus. Wien: Breitenstein.

Kortmann, M. (2007). Die Quadratur des Kreises. Johann Valentin Andreaes' 'Christianopolis'. In: http://edoc.sub.uni-hamburg.de/hsu/volltexte/2008/1340/(abgerufen: 20.08.2016)

Marx, K. (1977): Das Kapital Bd. 1, MEW, Bd. 23. Berlin: Dietz.

Wollgast, S. (1988): Johann Valentin Andreae. Versuch eines Überblicks. In: Ders.: Philosophie in Deutschland zwischen Reformation und Aufklärung 1550 - 1650. Berlin: Akademie Verlag: S. 263 345.

Wudmann, M. (2008): Wege aus der Krise. Johann Valentin Andrae und Johann Amos Comenius. In: Studia Comeniana et historica. Band XXXVIII: Studien zu Comenius und zur Comeniusrezeption in Deutschland. Uherský Brod: Muzeum J.A. Komenského: S. 93-113.

\section{Über die Autoren}

Anja Franz, M.A.: Promovendin und Dozentin am Lehrstuhl für Internationale und Interkulturelle Bildungsforschung der Otto-von-Guericke-Universität Magdeburg, Forschungsschwerpunkte: Bildung und soziale Ungleichheit, Hochschulforschung. Kontakt: anja.franz@ovgu.de

Dr. Dietrich-Eckhard Franz: Philosophiehistoriker, nach Philosophiestudium an der HumboldtUniversität Berlin Promotion an der Friedrich-Schiller-Universität Jena, Forschungsschwerpunkt: europäische Utopiegeschichte.

i $\quad$ Erstausgabe: Andreae, J.V. (1619): Republicae Christianopolitanae Descriptio. Argentorati: Sumptibus haeredum Lazari Zetzneri. Neuere Ausgaben: Biesterfeld, W. (Hg.) (1975): J. V. Andreae: Christianopolis. Stuttgart: Reclam-Verlag und Andreae, J.V. (1977): Christianopolis. Utopie eines christlichen Staates aus dem Jahre 1619. Leipzig: Koehler \& Amelang. Zu Leben und Werk Andreaes vgl. u.a. Andreae (1799), Wollgast (1988), Hoßbach (1977) sowie Dülmen (1978).

ii Man ist hier an W. Shakespeare erinnert, dessen Charakterisierung der Macht des Goldes in "Timon of Athens" Marx so treffend fand, dass er sie in das entsprechende Kapitel des "Kapital" aufnahm (vgl. Marx 1977: 145f.). 